\title{
An EPMA Study of the Soft Fe La-Lß X-ray lines in Fe-silicide, Olivine and Fe- sulfide Minerals by SXES and WDS
}

\author{
Aurélien $\operatorname{Moy}^{1 *}$, Anette von der Handt ${ }^{2}$ and John Fournelle ${ }^{1}$ \\ 1. Department of Geoscience, University of Wisconsin, Madison, WI 53706, USA. \\ 2. Department of Earth Sciences, University of Minnesota, Minneapolis, MN 55455, USA. \\ * Corresponding author: amoy6@wisc.edu
}

The recent development of the Soft X-ray Emission Spectrometer (SXES) allows the simultaneous acquisition of the whole soft X-ray spectrum, like the traditional SiLi or SDD EDS. This type of detector, consisting of a variable spacing diffraction grating, and a Peltier-cooled CCD camera [1], combines a spectral resolution comparable to the wavelength-dispersive spectrometers (WDS) $-4.5 \mathrm{eV}$ for the $\mathrm{Mg} \mathrm{K} \alpha \mathrm{X}$-ray line using the JEOL JS2000 extended range grating-to a detection efficiency close to the one that can be achieved with EDS. The typical energy range of acquisition is between few hundreds of eV to few $\mathrm{keV}$ (e.g., from $240 \mathrm{eV}$ to $2800 \mathrm{eV}$ for the JEOL JS2000 extended range grating) allowing the simultaneous measurements of most of the light elements useful for geologists using the $\mathrm{K}$ or L X-ray lines. For elements heavier than $\mathrm{Cl}$, the L or M X-ray lines should preferentially be used because the energy of the $\mathrm{K}$ lines is generally outside the measured energy range of the used diffraction grating, at first order of diffraction. However, the behavior of the characteristic L $\alpha$ and L $\beta$ X-ray lines of the first-row transition metals are not well known because complex chemical and physical effects can modify their properties. Indeed, the L $\alpha$ and $\mathrm{L} \beta$ X-ray lines of those elements are due to an electron transition from the partially filled $3 \mathrm{~d}$ electron shell which is subject to strong bonding effects with the electrons of the neighboring atoms. As a result, the outer electronic shells are modified by these bonding effects and then are the properties of the associated electrons (binding energy, transition probabilities, ...). Similar effects arise for the rare earth elements but with the M $\alpha$ and M $\beta$ X-ray lines. Because of these bounding effects, electron involved in a transition with the valence shells or an outer partially filled electron shell will produce X-ray lines whose shape and energy position may change depending on coordination types and/or oxidation states of the studied element [2]. These effects have advantages and drawbacks. The main advantage is that the chemical state of the elements can easily be studied using the SXES, or a WDS with the appropriate monochromator crystal, by comparing the shape and peak position of the L X-ray lines with spectra from standards of known composition, allowing the determination of the oxidation state or crystal structure of the studied material. However, the main drawback is that the atomic parameters associated with these soft X-ray lines, such as the mass absorption coefficient (MAC), the fluorescence yield, or the Coster-Kronig factor, are changing from one chemical state to another [3,4]. Therefore, quantitative results cannot be obtained using the traditional methods because accurate knowledge of these atomic parameters is essential to run the ZAF or phi- $\rho-z$ matrix correction algorithms.

We propose here a method to determine the Fe concentration in Fe-bearing mineral families using simple, platform/spectrometer-independent calibration curves that can directly be applied to the majority of electron microprobes currently in use. The calibration curves were obtained by recording the spectrum of the Fe L $\alpha$ and L $\beta$ X-ray lines on a set of Fe standards of well-known composition and by calculating the total recorded X-ray intensity corresponding to the area of these lines. A k-ratio can then be obtained, relative to the area intensity measured on a pure Fe standard and can be plotted as a function of the Fe composition. This method of using the peak area instead of the peak maximum, has 
the advantage to produce a k-ratio value that is independent of the spectrometer detection efficiency. The instrumental dependency that remains is due to the takeoff angle of the spectrometer. Hence, the only variables between calibration curves are the accelerating voltage (each one requires a different calibration curve) and the spectrometer takeoff angle. In our work, and for most electron microprobes, the takeoff angle is $40^{\circ}$. Therefore, the calibration curves obtained can be used for quantification purpose regardless of the spectrometer or instrument employed and without the need to "rescale" them.

We applied our method to the quantitative analysis of three different set of materials: iron-silicides, olivines and iron-sulfides. Figure 1 shows the area k-ratios measured on Fe-silicide standards by four different spectrometers-one SXES and three WDSs with different Rowland circles-on two different electron microprobes, as well as a set of olivine standards using five WDSs and one SXES. The experimental data acquired with the different spectrometers agree well with each other for each set of materials. The experimental area k-ratios vary smoothly with the Fe concentration allowing each data set to be fitted by a polynomial function with a good coefficient of determination. The calibration curves have been used to quantify Fe in unknown Fe-silicides and olivines at $7 \mathrm{kV}$ using the Fe L $\alpha$-L $\beta$ X-ray area intensity with good accuracy. The method was applied a third time, on a set of Fe-sulfide standards and led to another calibration curve that can be used to quantify Fe in this family of minerals (Fig. 2). It is worth mentioning that a calibration curve can also be derived using the maximum intensity of the $\mathrm{Fe}$ L $\alpha$ X-ray line, but such a curve must be "rescaled" on each instrument as it is dependent on the spectrometer used to acquire it. These two methods, the area and peak maximum calibration curves, provide an easy and accurate way to quantify Fe in families of Fe-bearing minerals using the L $\alpha$-L $\beta$ Xray lines. Further work is being investigated to extend these methods to other mineral families and more elements (such as $\mathrm{Cu}$ or $\mathrm{Ni}$ ) [5].

\section{References:}

[1] M Terauchi, et al, Microsc. Microanal. 17 (S2) (2011), p. 604.

[2] M Fialin et al, Mineral. Mag. 75 (2) (2011), p. 347.

[3] X Llovet et al, Microsc. Microanal. 22 (2016), p. 1233.

[4] B Buse and S Kearns, Microsc. Microanal. 24 (2018), p. 1.

[5] Support for this research came from the National Science Foundation: EAR13-37156 (JHF), EAR1554269 (JHF) and EAR-1625422 (AVDH).

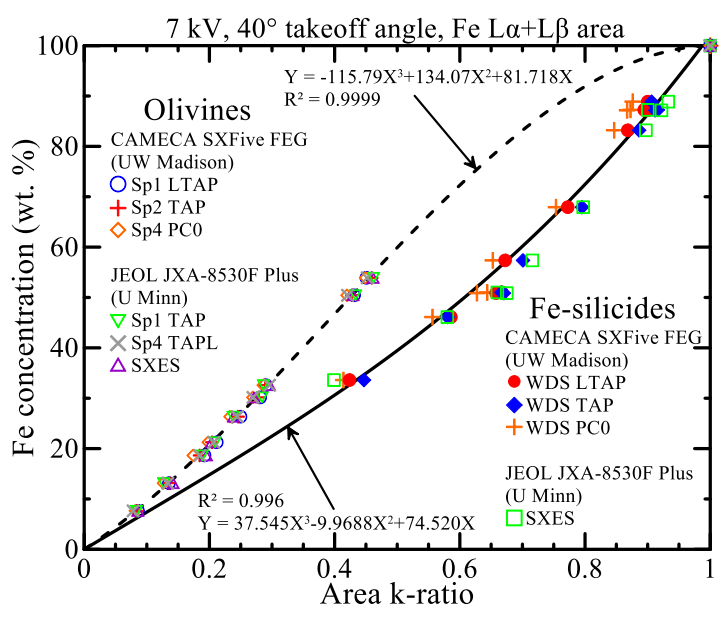

Figure 1. Fe L $\alpha$-L $\beta$ area k-ratios measured on Fesilicide and olivine standards and fitted with polynomial functions to derive calibration curves.

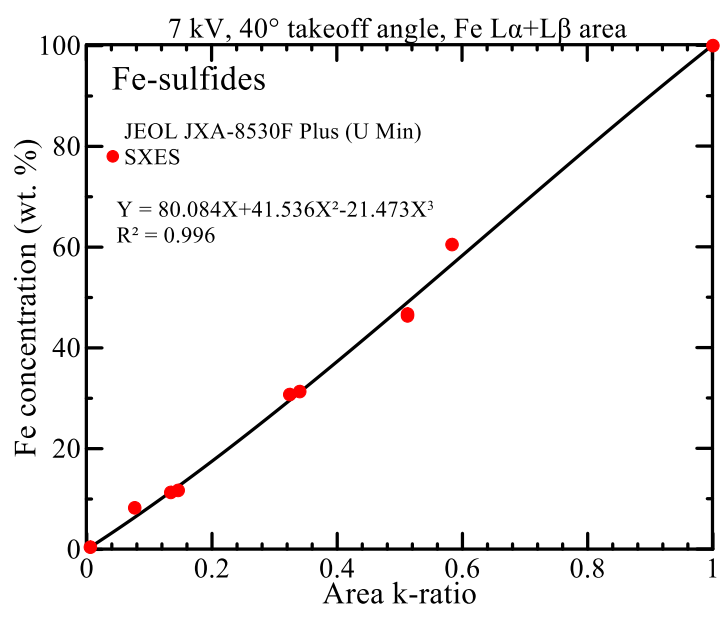

Figure 2. Calibration curve obtained at $7 \mathrm{kV}$ using the area of the Fe L $\alpha$-L $\beta$ X-ray line on Fesulfide standards. k-ratios are relative to pure Fe. 